\title{
Decoupling Eye-Specific Segregation from Lamination in the Lateral Geniculate Nucleus
}

\author{
Andrew D. Huberman, ${ }^{1}$ David Stellwagen, ${ }^{2}$ and Barbara Chapman ${ }^{1}$ \\ ${ }^{1}$ Center for Neuroscience, University of California, Davis, California 95616, and ${ }^{2}$ Department of Psychiatry and Behavioral \\ Sciences, Stanford University School of Medicine, Palo Alto, California 94304
}

To determine whether there is a critical period for development of eye-specific layers in the lateral geniculate nucleus (LGN), we prevented the normal segregation of retinogeniculate afferents and then allowed an extended period of time for recovery. After recovery, both anatomy and physiology revealed strictly nonoverlapping territories of input from the two eyes. However, the normal stereotyped pattern of eye-specific afferent and cellular layers never developed. Instead, the eye-specific territories of afferent input emerged as variable and disorganized patches with no corresponding interlaminar spaces in the LGN. These findings reveal a critical period for coordinating the development of three processes in the LGN: the segregation of afferents from the two eyes, the spatial organization of those afferents into layers, and the alignment of postsynaptic cytoarchitecture with the afferent inputs. We also assessed the physiological consequences of preventing normal lamination and found normal single-cell responses and topographic representation of visual space in the LGN. Clusters of ON-center and OFF-center LGN cells were segregated from one another as in normal animals. However, the organization of ON and OFF sublaminas in the treated animals was disrupted.

Key words: visual system; development; lateral geniculate nucleus; activity; lamination; eye-specific; critical period
The adult LGN is characterized by its stereotyped organization into discrete eye-specific layers. These layers are evident both as nonoverlapping regions of afferents from the two eyes and as corresponding cellular laminas formed by LGN neurons (Jones, 1985). The mechanisms underlying the development of the afferent and cellular LGN layers are not well understood.

During normal development, the retinogeniculate afferents from the two eyes initially overlap before gradually segregating into eye-specific layers (Fig. 1A,B) (Linden et al., 1981; Cucchiaro and Guillery, 1984; Hutchins and Casagrande, 1990; Penn et al., 1998; Hahm et al., 1999). Previous experiments suggest that the formation of afferent layers in the LGN occurs via a competition between the two eyes for postsynaptic space. If one eye is removed before eye-specific segregation, axons from the remaining eye expand into regions of the LGN normally occupied only by the other eye (Lund et al., 1973; Rakic, 1981; Guillery et al., 1985a; Sretavan and Shatz, 1986; Garraghty et al., 1988; Thompson et al., 1993). Furthermore, this competition between the two eyes in normal development is mediated by spontaneous retinal activity. If activity from both eyes is pharmacologically silenced, axons from the two eyes do not segregate (Fig. $1 C-E$ ) (Shatz and Stryker, 1988; Penn et al., 1998; Rossi et al., 2001). If activity is silenced in one eye, there is an expansion of the territory of the active untreated eye at the expense of the projection from the

Received April 15, 2002; revised Aug. 13, 2002; accepted Aug. 23, 2002.

This work was supported by National Institutes of Health (NIH) Grant EY11369 (B.C.), Core Grant EY12576, and an NIH Systems Neuroscience Training Fellowship (A.D.H.). We thank B. Reese for advice on retinal histology; R. Berman and B. Barres for use of their imaging facilities; S. Sabo for advice on image quantification; A. Haines and P. Nguyen for expert technical assistance; W. Usrey, M. Sceniak, O. Collins, and $\mathrm{H}$. Alitto for assistance with white-noise receptive field mapping; and L. Stone for helpful comments on this manuscript.

Correspondence should be addressed to Dr. Barbara Chapman, Center for Neuroscience, 1544 Newton Court, Davis, CA 95616. E-mail: bxchapman@ ucdavis.edu.

Copyright () 2002 Society for Neuroscience $\quad 0270-6474 / 02 / 229419-11 \$ 15.00 / 0$ inactive eye (Penn et al., 1998; Stellwagen and Shatz, 2002). Conversely, if the level of activity in one eye is elevated, there is an expansion of the territory of the more active eye at the expense of the normally active untreated eye (Stellwagen and Shatz, 2002).

The formation of eye-specific cellular layers in the LGN is proposed to be driven by the retinal afferents themselves. Normally, the development of cellular layers occurs after afferents from the two eyes segregate (Linden et al., 1981; Cucchiaro and Guillery, 1984; Hutchins and Casagrande, 1990). If the pattern of retinogeniculate afferents is abnormal, the cytoarchitecture of the LGN directly reflects these abnormal inputs. For example, in monocularly or binocularly enucleated animals (Brunso-Bechtold and Casagrande, 1981; Rakic, 1981; Guillery et al., 1985a,b; Sretavan and Shatz, 1986; Garraghty et al., 1988; Morgan and Thompson, 1993), eye-specific cellular laminas also do not develop. Moreover, in coat-color mutants, in which the density of the ipsilateral-eye projection to the LGN is reduced, the cellular laminas mirror the abnormal topography of the retinal projections (Guillery, 1969, 1971).

Our experiment was designed to further study the developmental interaction between afferents from the two eyes and between these retinal afferents and the cytoarchitecture of the LGN. Specifically, we sought to determine the role of spontaneous retinal activity in these interactions and to look for a critical period for development of LGN lamination. To do so, we prevented the normal eye-specific segregation of retinogeniculate inputs by silencing spontaneous retinal activity in both eyes and then allowed these animals an extended period of recovery, during which spontaneous retinal activity returned to normal. We then assessed the effects of this manipulation on the pattern of retinogeniculate inputs, the cytoarchitecture of the LGN, and the physiology of LGN neurons, including their receptive field properties and spatial organization. 


\section{MATERIALS AND METHODS}

Animals. Timed pregnant Fitch-coat ferrets were obtained from Marshall Farms (New Rose, NY). All experimental procedures were performed in accordance with approved animal use protocols at University of California Davis. Postnatal day 0 (P0) corresponds to the day of birth. Animals in all treatment groups gained weight normally throughout the course of the study (Chapman and Godecke, 2000).

In vivo intravitreal application of epibatidine. Surgical and drug injection procedures were similar to those described previously (Penn et al., 1998; Chapman, 2000; Stellwagen and Shatz, 2002). Briefly, every $48 \mathrm{hr}$ from P1 until P10, animals were anesthetized with inhalant isofluorane and 1-2 $\mu \mathrm{l}$ of epibatidine $\mathrm{HCl}$ (1 mM dissolved in sterile saline) (dose of $1 \mu \mathrm{l}$ on P1, increased by $0.25 \mu \mathrm{l}$ for each subsequent injection; Sigma, St. Louis, MO), or an equivalent volume of control solution (sterile saline) was injected into the vitreous humor of each eye at a rate of $0.5 \mu \mathrm{l} / \mathrm{min}$ using a 33 gauge needle attached to a Hamilton (Reno, NV) microsyringe.

Labeling of retinogeniculate afferents and cytoarchitecture in the LGN. Ferrets received intravitreal injections of cholera toxin- $\beta$ subunit $(\mathrm{CT} \beta)$ conjugated to fluorescein (green label) in the right eye and CT $\beta$ conjugated to rhodamine (red label) into the left eye (3-8 $\mu$ l depending on the age of the animal; $0.5 \%$ in sterile saline; List Biochemical) (CT $\beta$ has no biological activity). One day later, animals were transcardially perfused with $4 \%$ paraformaldehyde (ages in text correspond to age at which animals were killed), and tissue was postfixed overnight and sectioned at $50 \mu \mathrm{m}$. After imaging of retinal afferents (see below), LGN sections were dehydrated and stained with Thionin $(0.45 \%)$ to reveal cytoarchitecture.

Retinal histology. Retinal flatmounts were prepared by dissecting out the retina whole from the eyecup and placing four relieving cuts along the major axis, radial to the optic nerve. The entire retina was stained with 4',6'-diamidino-2-phenylindole (Vector Laboratories, Burlingame, CA) to reveal cell nuclei; CT $\beta$ label allowed identification of nuclei and, for ganglion cells, an axon. For retinal cross sections, eyes were hemisected, embedded, sectioned at $15 \mu \mathrm{m}$, and processed with Thionin.

Image quantification. All images were digitally acquired with a CCD camera (SPOT Diagnostic); universal gains were established for each label at a given magnification. Raw images of the LGN were imported to PhotoShop (Adobe Systems, San Jose, CA) and cropped to exclude the optic tract and medial intralaminar nucleus. Images were then thresholded to $30 \%$ above background (designated as a nonretinorecipient portion of the tissue slice $1 \mathrm{~mm}$ lateral to the midline of the thalamus). The $30 \%$ value is based on previous studies (Stellwagen and Shatz, 2002) and evaluation of signal:noise in tissue from animals of different ages. Thresholded images were then set to black $(<30 \%$ above threshold) or white ( $>30 \%$ above threshold). Measurements of the area of the LGN occupied by the contralateral or ipsilateral eye projections were calculated by automatically selecting all white pixels within the image frame (Scion Image; Scion Corp., Frederick, MD). Measurements of overlap were calculated by multiplying the thresholded image of the contralateral eye inputs to the LGN with the thresholded image of the ipsilateral eye inputs to the same LGN. In the resulting image, white pixels thus correspond only to locations at which the contralateral and ipsilateral afferents were both present; overlap area was calculated by automatically selecting all of the white pixels within the image frame (Scion Image). To quantify the extent of the ipsilateral projection to the LGN, the boundary of the total ipsilateral projection (including $\mathrm{A}, \mathrm{A} 1$, and $\mathrm{C}$ laminas in normal animals and all ipsilateral eye label in epibatidine-recovery animals) was delineated by connecting the edges of the outermost ipsilateral projections with straight lines; then the total area contained within the resulting polygon was divided by the total area of the LGN in that section. Three to six sections through the middle $200-300 \mu \mathrm{m}$ portion of the LGN were analyzed for each animal (depending on the age of the animal). LGNs from animals in which retinal ganglion cell or retinogeniculate labeling appeared incomplete were excluded from all analyses.

Measurements of both total cell and ganglion cell density were performed from matched locations in the central and peripheral retina for all four retinal quadrants. Morphological criteria described previously were used to identify different cell types (Henderson et al., 1988; Stellwagen and Shatz, 2002). Quantification was limited to retinas P25 and older (P25+) (after ganglion cell genesis and apoptosis in the ferret retina is complete) (Henderson et al., 1988; Reese et al., 1994; Cusato et al., 2001). The entire retinas from ferrets of all ages were carefully inspected for damage.

Preparation of photomicrographs. Images were imported to PhotoShop (Adobe Systems) for cropping, resizing, and alignment. In some cases, artifact was removed from outside the boundaries of the LGN.
Imaging of spontaneous retinal activity. Imaging of spontaneous retinal activity was performed according to protocols described previously (Feller et al., 1996; Penn et al., 1998; Stellwagen et al., 1999; Wong et al., 2000; Stellwagen and Shatz, 2002). Ferrets were deeply anesthetized with halothane and then decapitated. Retinas were then rapidly dissected from the eye and incubated in $10 \mu \mathrm{M}$ fura-2 AM (Molecular Probes, Eugene, OR) in artificial CSF [ACSF (in mM): $119 \mathrm{NaCl}, 2.5 \mathrm{KCl}, 1.3$ $\mathrm{MgCl}_{2}, 1 \mathrm{KH}_{2} \mathrm{PO}_{4}, 2.5 \mathrm{CaCl}_{2}, 26.2 \mathrm{NaHCO}_{3}$, and 11 D-glucose] containing $1 \%$ DMSO and $0.02 \%$ pluronic acid for $4-8 \mathrm{hr}$ in an oxygenated chamber at $28^{\circ}$. Optical recording was performed on an inverted microscope (Diapshot 300; Nikon, Tokyo, Japan) using a $5 \times$ objective (to assess activity across an entire quadrant of the retina) or $40 \times$ objective (to assess correlations between individual neighboring cells; average of 20 cells per field). Retinas were perfused continuously with $32^{\circ}$ ACSF throughout the imaging session. In some cases, retinas were perfused with $100 \mathrm{~nm}$ epibatidine (in ACSF) during the imaging session. Images were acquired with a pentamax cooled CCD camera (Princeton Instruments, Trenton, NJ), using Metamorph software (Universal Imaging, West Chester, PA). Difference images were calculated from an initial background frame. Fluorescence data are presented as $\Delta F / F$ versus time, where $F$ is the amount of direct current fluorescence corrected for bleaching and $\Delta F$ is the deviation from this baseline. Wave frequency was determined by dividing the total number of $\geq 1 \% \Delta F / F$ events/time. Wave domain sizes were calculated by measuring the contiguous extent of an area where a propagating $>1 \% \Delta F / F$ event occurred.

Extracellular recordings of visual responses in the LGN. LGN recordings were performed using protocols described previously (Stryker and Zahs, 1983; Zahs and Stryker, 1985; Chapman, 2000; Chapman and Godecke, 2000). Ferrets were anesthetized using a mixture of acepromazine (0.4 $\mathrm{mg} / \mathrm{kg}$ ) and ketamine $(40 \mathrm{mg} / \mathrm{kg}$, i.m.) and placed in a modified kitten stereotax. Animals were intubated and anesthesia was maintained using $1-2 \%$ inhalant isoflurane in oxygen (volume and rate to maintained peak inspiratory pressure at $1.5 \mathrm{kPa}$ and end-tidal carbon dioxide at $3.5-5 \%$ ). End-tidal carbon dioxide, core body temperature, and electrocardiogram were monitored throughout the experiment. In cases in which retinotopy was mapped, animals were paralyzed with vecuronium bromide $(0.2$ $\mathrm{mg} \cdot \mathrm{kg}^{-1} \cdot \mathrm{hr}^{-1}$, i.v.) and ventilated mechanically. A $4 \times 4 \mathrm{~mm}$ craniotomy was made over the LGN, lacquered tungsten electrodes (Micro Probe, Potomac, MD) were advanced through the depth of the LGN using a microdrive, and visual responses were recorded every $100 \mu \mathrm{m}$. To map retinotopy and the organization of ON- and OFF-center cells, LGN activity was assessed with an audio monitor and oscilloscope, and receptive fields were mapped onto a tangent screen using a handheld light. Electrode penetrations were spaced in a $300 \mu \mathrm{m}$ grid across the rostrocaudal and mediolateral extent of the LGN. To obtain peristimulus time histograms (PSTHs) and receptive field maps, visual stimuli were created using a VSG 2/5 visual stimulator (Cambridge Research Systems, Rochester, UK) and were displayed on a Sony monitor with a mean luminance of 40-50 candelas $/ \mathrm{m}^{2}$. Single units were discriminated, and spike times and waveforms were recorded using a Spike 2 system (Cambridge Electronic Design, Cambridge, UK). Receptive field maps were calculated by reverse correlation from responses to white noise (m-sequence) stimuli (Reid et al., 1997). The white noise stimulus consisted of a $25 \times 25$ grid of squares that were white or black $50 \%$ of the time, as determined by an $\mathrm{m}$-sequence of length $2^{15}-1$. The stimulus was updated every $7.14 \mathrm{msec}$. PSTHs show averaged responses from 40 presentations of an alternating black/white disk on a neutral gray background.

At the end of the experiment, animals were injected intraocularly with anterograde tracers to visualize retinogeniculate afferents (see above); the animals were killed $24 \mathrm{hr}$ later, and LGN and retinal tissue was harvested for anatomical assessment of the epibatidine treatment (see above) and reconstruction of the electrode tracks. Recording positions along electrode tracks were reconstructed by matching the locations where visually responsive neurons were first and last encountered with the dorsal and ventral anatomical borders of LGN. Positions along the tracks were also verified by matching where the physiological responses changed from one eye to the other, with the borders of eye-specific retinogeniculate $\mathrm{CT} \beta$-fluorescein/rhodamine.

\section{RESULTS \\ Activity blockade prevents segregation of eye-specific inputs to the LGN}

In normal P1 ferrets $(n=6)$, ganglion cell axons from the two retinas overlap extensively (Fig. $1 A$ ) (Linden et al., 1981; Cuc- 

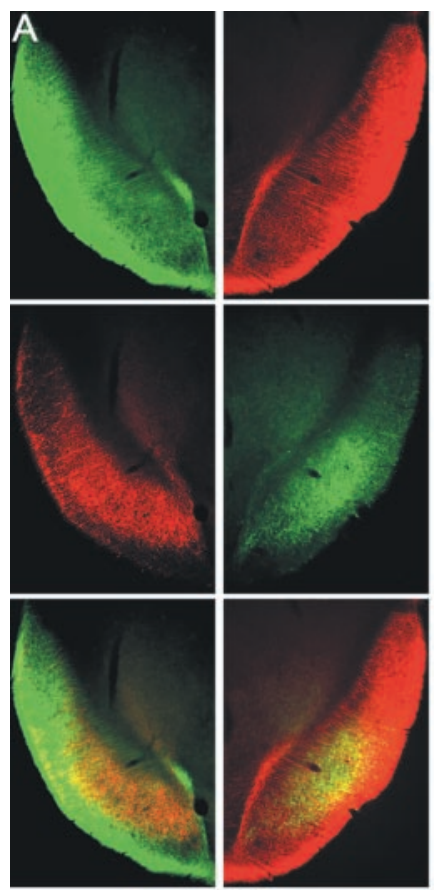

C

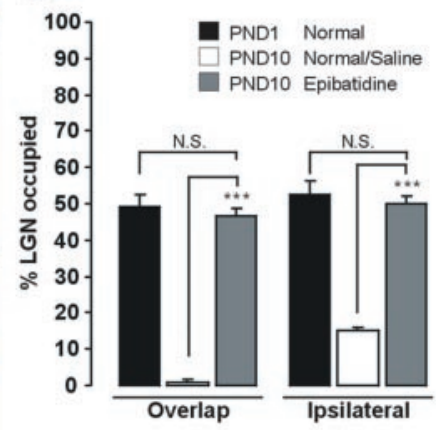

D
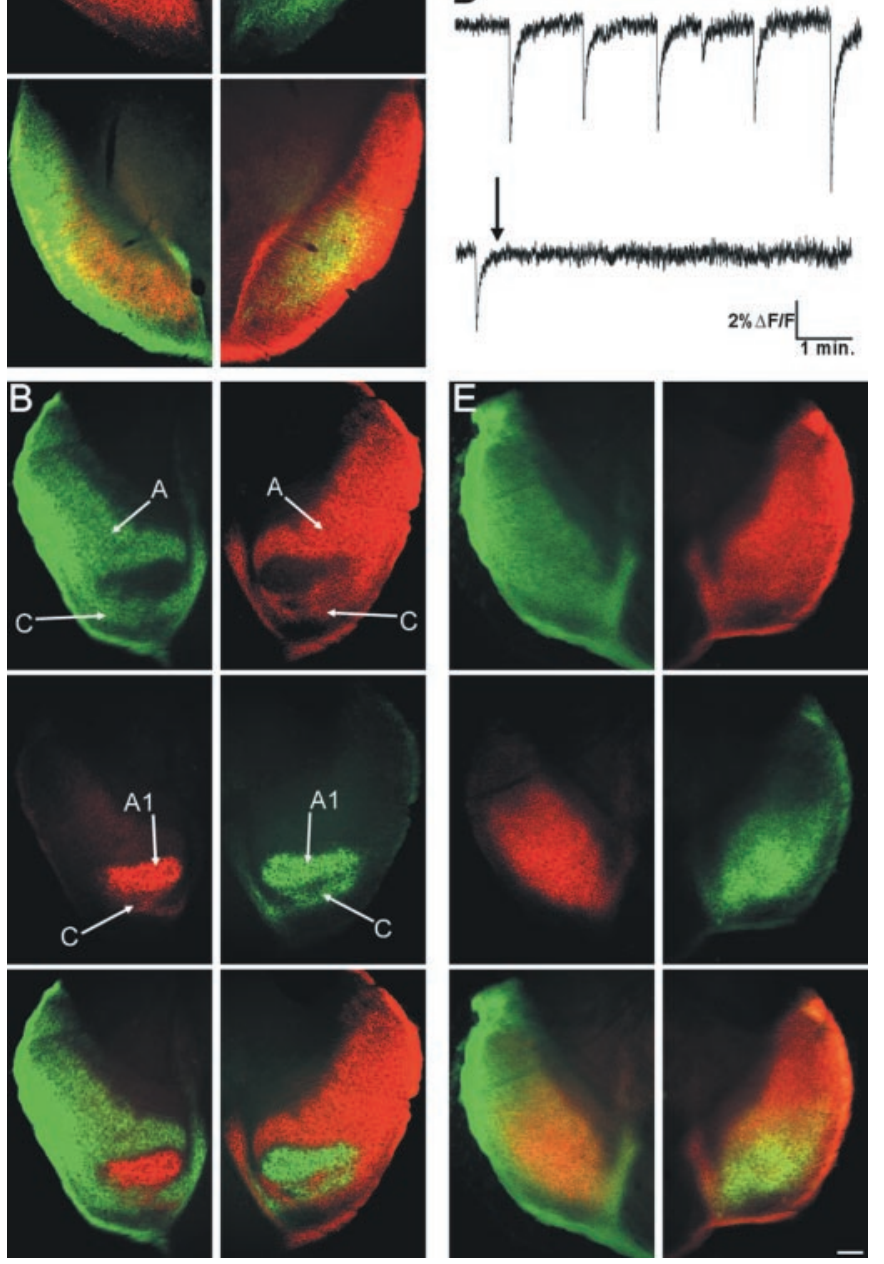

Figure 1. Effect of epibatidine on spontaneous retinal activity and development of eye-specific segregation in the LGN. $A, B, E$, Contralateral (top panels) and ipsilateral (middle panels) retinal inputs to the LGN and their merged representation (bottom panels) from a normal P1 ferret $(A)$, a normal P10 ferret $(B)$ (arrows indicate lamina $A, A 1$, and $C$ ), and a P10 ferret that received intravitreal injections of epibatidine every $48 \mathrm{hr}$ from $\mathrm{P} 1$ to $\mathrm{P} 10(E)$. Tissue sections are in the horizontal plane; rostral is to the top, and medial is to the center of each panel. Scale bar: $A, 50 \mu \mathrm{m} ; B, E$, $75 \mu \mathrm{m}$. $C$, Percentage of LGN area occupied by overlapping projections from both eyes (overlap) or the ipsilateral eye on P1 ( $n=6$ LGNs) and P10 after normal/saline or epibatidine treatment $(* * * p<0.0001)$ (N.S., not significantly different) (overlap, $p=0.5$; ipsilateral, $p=0.49$; $t$ test; $n=6$ normal $/ 6$ saline, $n=12$ epibatidine). $P N D$, Postnatal day. $D$, Epibatidine abolishes early spontaneous retinal activity. Top trace, Normal P5 ferret retina showing spontaneous periodic increases in intracellular $\mathrm{Ca}^{2+}$ (downward deflections); bottom trace, $\mathrm{P} 5$ ferret retina showing one initial $\mathrm{Ca}^{2+}$ wave and then complete, sustained elimination of wave activity after application of $100 \mathrm{~nm}$ epibatidine (arrow). chiaro and Guillery, 1984; Hutchins and Casagrande, 1990; Penn et al., 1998; Hahm et al., 1999), with $\sim 50 \%$ of the LGN receiving inputs from both eyes (Fig. 1C). By P10, axons from the two eyes are segregated (Fig. 1B) (Linden et al., 1981; Cucchiaro and Guillery, 1984; Hutchins and Casagrande, 1990; Penn et al., 1998; Hahm et al., 1999; Chapman, 2000) with $<1 \%$ of the LGN receiving overlapping contralateral and ipsilateral retinal axons (Fig. 1C). The segregation of eye-specific inputs to the LGN can be prevented by silencing spontaneous retinal activity (Fig. 1C,E) (Penn et al., 1998; Rossi et al., 2001). In P1-P10 ferret retinas, spontaneous retinal activity is completely silenced by application of epibatidine (Fig. 1D) (Penn et al., 1998; Stellwagen et al., 1999). Immediately after the binocular epibatidine treatment from P1 to P10 $(n=6)$, inputs from the two eyes overlap throughout $\sim 50 \%$ of the LGN (Fig. $1 E, C$ ), similar to the overlapping pattern seen in normal $\mathrm{P} 1$ ferrets (Fig. $1 A, C$ ) and unlike the segregated pattern seen in control P10 ferrets $(n=3$ untreated; $n=4$ saline injected) (Fig. $1 B, C$ ). Two features underlie the overlap seen in both normal P1 ferrets and P10 epibatidinetreated ferrets: the contralateral eye projects to the entire retinorecipient area of the LGN (Fig. $1 A, E$, top panels), and the ipsilateral retinal projection is significantly expanded (Fig. $1 A, E$, middle panels, $C)$.

\section{Recovery restores eye-specific segregation but not afferent lamination}

To determine whether there is a critical period for the development of the normal pattern of eye-specific inputs to the LGN, a group of ferrets received binocular intravitreal injections of epibatidine every $48 \mathrm{hr}$ from P1 to P10 $(n=15)$ but were then allowed to survive until P25 $(n=8)$, P36 $(n=4)$, or P65-P100 $(n=3)$, at which time retinogeniculate afferents were visualized. In control ferrets $\mathrm{P} 25$ and older $(\mathrm{P} 25+)$ that received either no treatment $(n=6)$ or binocular intravitreal injections of saline from $\mathrm{P} 1$ to $\mathrm{P} 10(n=8)$, retinogeniculate afferents are completely segregated into eye-specific A and A1 laminas (overlap area of $<1 \% ; n=22$ LGNs) as well as into ON and OFF sublaminas (see Figs. 2A, 4B,C) (Linden et al., 1981; Stryker and Zahs, 1983; Cucchiaro and Guillery, 1984; Zahs and Stryker, 1985; Hutchins and Casagrande, 1990; Hahm et al., 1999). The pattern of retinal inputs to the LGN of the epibatidine-treated animals that were allowed to recover until $\mathrm{P} 25+$ (hereafter referred to as epibatidine-recovery animals) is dramatically different (compare Figs. $2 A, 4 B, C$ with Figs. $2 B, 4 F, G, J, K, N, O)$. Although inputs from the two eyes achieved normal levels of segregation in these animals (overlap area of $<1 \%$; $n=26$ LGNs; $p=0.48$ recovery animals vs controls), the pattern of eye-specific inputs is highly aberrant, with multiple ipsilateral projections of various shapes, positions, and sizes distributed over a significantly greater-thannormal extent of the LGN (Fig. 2C). These abnormal projections often extend into the region of the nucleus normally occupied only by afferents from the contralateral eye (compare Fig. $2 A$ with $2 B$ and Fig. $4 B, C$ with $4 F, G, J, K, N, O)$ and can even be displaced to the medial border of the nucleus (Fig. $2 B$, arrows in middle and bottom panels).

Discontinuities in the contralateral-eye and ipsilateral-eye layers have been shown previously to accompany reductions in the density of the ipsilateral retinogeniculate projection in coat-color mutants (Guillery, 1969, 1971). However, a simple reduction in the ipsilateral projection cannot explain the multiple eye-specific patches seen in the epibatidine-recovery animals, because the area 

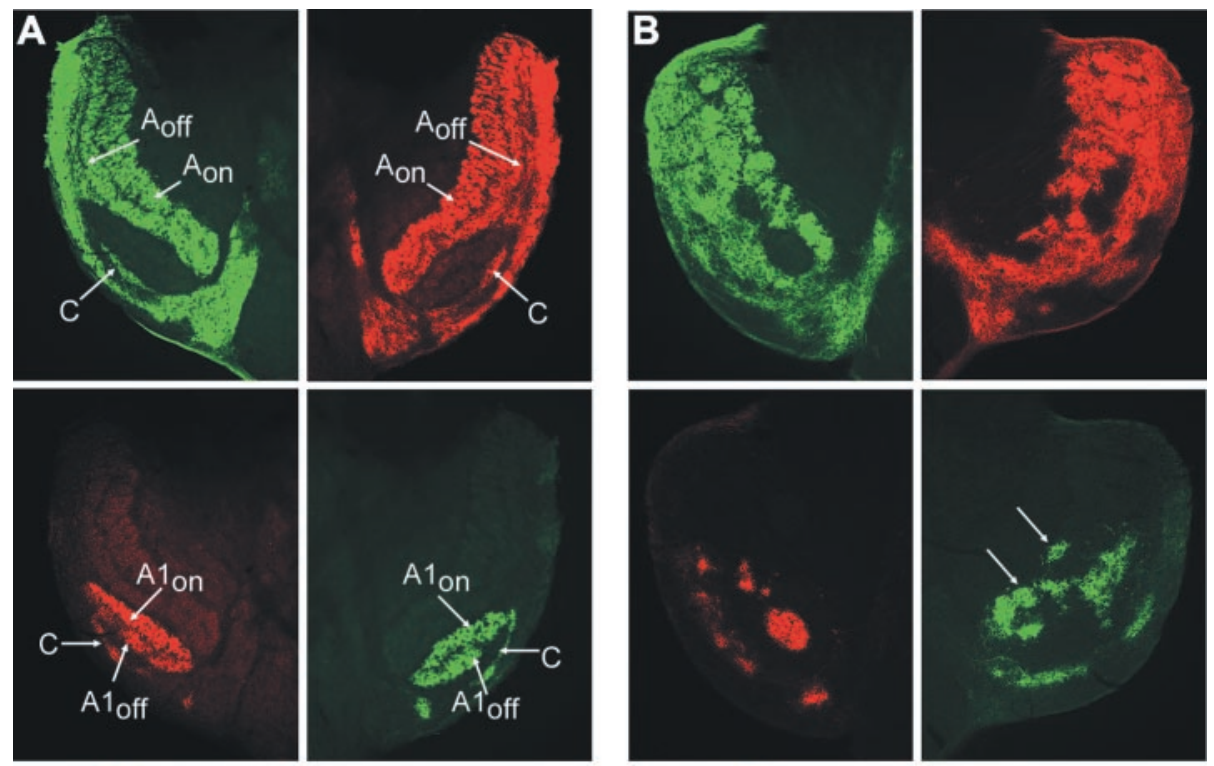

Figure 2. Intravitreal epibatidine from $\mathrm{P} 1$ to $\mathrm{P} 10$, followed by recovery, disrupts normal patterning of eye-specific laminas in the LGN. $A, B$, Contralateral (top panels) and ipsilateral (middle panels) retinal inputs to the LGN and their merged representation (bottom panels) from a P25 ferret treated with saline from P1 to P10 $(A)$ [arrows indicate ON and OFF sublaminas of laminas $\mathrm{A}\left(A_{\text {on }}, A_{\text {off }}\right)$ and $\mathrm{A} 1\left(A 1_{\text {on }}, A 1_{\text {off }}\right)$; C laminas are also seen] and a P25 ferret injected with epibatidine from P1 to P10 (B) (arrows indicate afferents from the ipsilateral retina that extend to ectopic locations along the medial border of the LGN). Tissue sections are in the horizontal plane; rostral is to the top, and medial is to the center of each panel. Scale bar, $100 \mu \mathrm{m}$. $C$, Quantification of the extent of ipsilateral retinal afferents across the LGN in the two treatment groups $(* * * p<0.0001)(t$ test; $n=11$ controls; $n=13$ epibatidine). $D$, Quantification of the LGN area occupied by ipsilateral retinal afferents in the two treatment groups $\left({ }^{*} p<0.05\right.$; $\left.{ }^{* *} p<0.01\right)(t$ test; $n=11$ control LGNs; $n=13$ epibatidine). PND, Postnatal day.
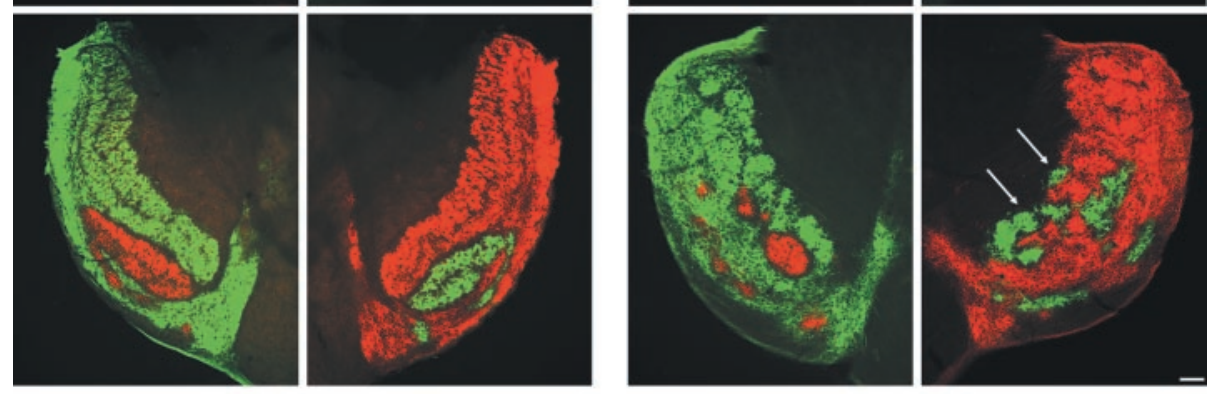

\section{C}

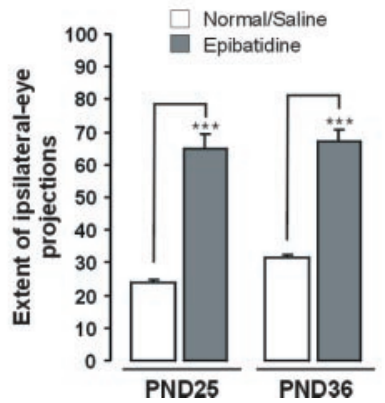

D

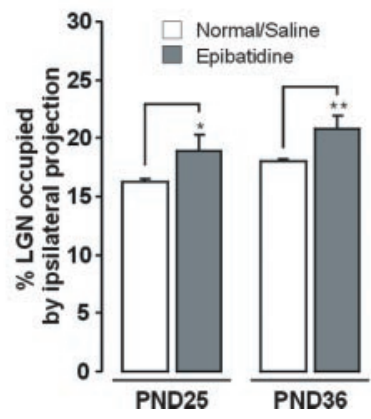

of the LGN occupied by the ipsilateral projections is not reduced but is in fact slightly increased relative to controls (Fig. 2D).

\section{Epibatidine treatment does not damage the retina, and spontaneous retinal activity re-emerges after the drug treatment}

Damage to the developing retina caused by the drug or tracer injections cannot explain the abnormal pattern of afferents seen in the epibatidine-recovery animals. Total retinal area does not differ between the treatment groups, and cell density is normal across all four quadrants of the treated retinas (total cell density, $100 \pm 1.4 \%$ of controls; ganglion cell density, $100 \pm 1.8 \%$ of controls; $n=8$ controls and $n=8$ epibatidine-treated retinas). The thickness of the nuclear and synaptic layers of the retina is also normal in the treated group (Fig. $3 A, B$ ). In addition, there are no ectopic patches in the retinogeniculate projection of the treated animals at P10 (Fig. 1E) or regions devoid of retinogeniculate afferents at any age after the drug treatment (Figs. $1 E$, $2 B, 4 F, G, J, K, N, O)$, either or both of which would be expected if lesions of the early postnatal retina had occurred (Jeffrey, 1985; Hanson and Reese, 1993). Deliberate needle-induced lesions to the retina performed on $\mathrm{P} 4$ or P34, followed by subsequent labeling of retinal ganglion cells on P36, confirm that after both long and short survival periods, needle-induced damage to the early postnatal ferret retina is easily detected (Fig. 3C,D). No such damage was evident in any of the animals included in this study.

Because cholinergic agents fail to suppress spontaneous retinal activity in ferret retinas older than P12 (Wong et al., 2000), it is unlikely that residual blockade of spontaneous retinal activity after cessation of the drug treatment could have contributed to the abnormal pattern of afferents seen in the epibatidine-recovery animals. In fact, in ferrets that received binocular injections of epibatidine every $48 \mathrm{hr}$ from P12 to P25, the LGN appeared normal (data not shown). However, to determine whether normal patterns of spontaneous retinal activity re-emerge after the epibatidine treatment, and to further determine that the drug and/or 

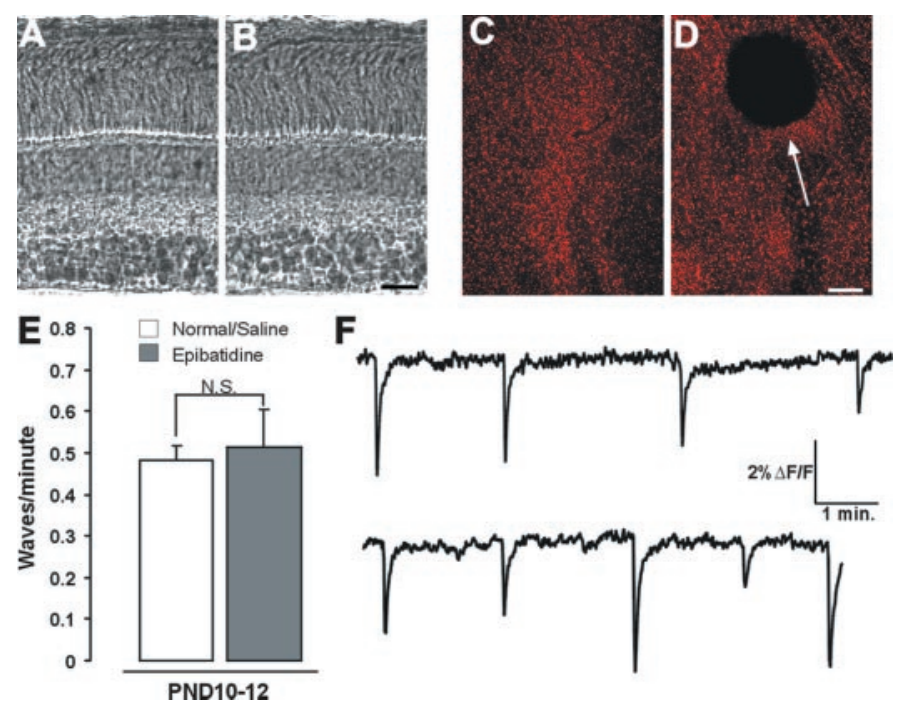

Figure 3. Epibatidine injections from P1 to P10 do not disrupt the structural integrity of the retina or the patterns of spontaneous retinal activity that re-emerge after treatment. $A, B$, Cross sections of the retina from a normal P36 ferret $(A)$ and a P36 ferret that was treated with epibatidine from P1 to P10 $(B)$. Scale bar, $50 \mu \mathrm{m}$. $C$, $D$, Lowmagnification view of the ventral retina from a P36 ferret that was treated with epibatidine from $\mathrm{P} 1$ to $\mathrm{P} 10(C)$ and a P36 animal that received a needle-induced lesion to the retina on P4 (D) (arrow indicates site of degeneration in the ganglion cell layer). CT $\beta$-rhodamine label of cell bodies is shown. Scale bar, $250 \mu \mathrm{m}$. $E$, Quantification of the frequency of retinal waves present in normal and epibatidine-treated retinas on P10P12 (N.S., not significant; $p=0.69)(t$ test; $n=12$ controls, $n=9$ treated retinas). $P N D$, Postnatal day. $F$, Examples of spontaneous retinal activity recorded from a normal (top trace) and epibatidine-treated (bottom trace) $\mathrm{P} 10-\mathrm{P} 12$ retina; values for each trace represent an area of the ganglion cell layer encompassing $\sim 20$ cells.

injection procedure did not render portions of the retina dead or permanently inactive, we analyzed patterns of spontaneous retinal activity (Feller et al., 1996; Penn et al., 1998; Stellwagen et al., 1999; Wong et al., 2000; Stellwagen and Shatz, 2002) present in control (untreated, $n=8$ retinas; saline, $n=9$ retinas) or epibatidine-treated ( $n=9$ retinas) P10-P12 ferret retinas that received injections every $48 \mathrm{hr}$ from $\mathrm{P} 1$ to P10. Fluorescence imaging of intracellular $\mathrm{Ca}^{2+}$ confirmed that by P10-P12, ganglion cells in the epibatidine-treated retinas exhibited highly correlated, periodic bouts of spontaneous activity with propagation patterns characteristic of normal P10-P12 "retinal waves." Low-magnification imaging across all four retinal quadrants confirmed that the spontaneous retinal activity that re-emerges from P10-P12 tiles the entire surface of the ganglion cell layer and is indistinguishable from that of normal or saline-treated controls in terms of frequency, duration, or amplitude (Fig. $3 E, F$ ). Wave domain size was also normal in the epibatidine-recovery retinas $\left(0.31 \pm 0.05 \mathrm{~mm}^{2} ; n=19\right.$ waves, vs $0.30 \pm 0.06 \mathrm{~mm}^{2}$ in control retinas; $n=19$ waves), indicating that retinal ganglion cells in both groups are well correlated in their firing.

\section{Epibatidine treatment followed by recovery abolishes the normal patterns of cellular lamination in the LGN}

In control ferrets, LGN cells form cytoarchitectural layers that lie in direct registration with the layers formed by the terminals of retinal afferents; they are concentrated into distinct $\mathrm{A}$ and A1 laminas, as well as $\mathrm{ON}$ and $\mathrm{OFF}$ sublaminas, each separated by a cell-sparse interlaminar space (Fig. 4A-D, arrowheads) (Linden et al., 1981; Stryker and Zahs, 1983; Cucchiaro and Guillery,
1984; Zahs and Stryker, 1985; Hutchins and Casagrande, 1990; Hahm et al., 1999). In contrast, the LGNs of the epibatidinerecovery animals completely lack normal patterns of cellular lamination (Fig. 4E,H,I,L,M,P). Clusters of cells, surrounded by cell-sparse regions, are occasionally visible, but comparison of these clusters with the pattern of retinogeniculate afferents in the same tissue sections reveals that they do not correspond to eyespecific termination zones of ganglion cell axons (for example, compare arrowheads in Fig. $4 E, F)$.

Although the spatial organization of afferent and cellular laminas seen in each LGN of control animals is remarkably stereotyped (Figs. 2A, 4A-D) (Linden et al., 1981; Stryker and Zahs, 1983; Cucchiaro and Guillery, 1984; Jones, 1985; Zahs and Stryker, 1985; Hutchins and Casagrande, 1990; Hahm et al., 1999), a striking feature of the pattern of retinogeniculate inputs of the epibatidine-recovery animals is their variability between animals (compare Figs. $2 B, 4 G-N$ ), between the two LGNs of the same animal (Fig. $4 M-P$ ), and even across short distances $(<100$ $\mu \mathrm{m}$ ) through the depth of the same LGN (Fig. $4 F, J$ ).

\section{Effects of early epibatidine treatment on visual responsiveness and functional organization of the LGN}

To determine whether disrupting the pattern of retinal afferent lamination alters the physiology of LGN neurons, we performed extracellular recordings in epibatidine-recovery animals. LGN neurons responded vigorously to visual stimuli, confirming that the drug treatment did not disrupt the overall health of the retinogeniculate pathway (epibatidine, $n=425$ recording sites, $n=4$ ferrets aged P52-P71; controls, $n=383$ recording sites, $n=$ 9 ferrets aged P37 to adult). All cells encountered were monocular, indicating that functional as well as anatomical segregation of eye-specific inputs to the LGN occurred after the termination of the epibatidine treatment. Cells in the LGN of the treated animals exhibited ON- or OFF-center responses typical of normal ferrets (Fig. 5) (Stryker and Zahs, 1983; Zahs and Stryker, 1985; Chapman and Gödecke, 2000). Both sustained (Fig. 5A,E,C,G) and transient (Fig. $5 B, F, D, H$ ) responses of both center types were present in both epibatidine- and saline-treated animals. The cells in the treated animals showed normal center-surround organization (compare Fig. $6 A-D$, saline treated, with Fig. $5 E-H$, epibatidine treated), typical of ferrets at this stage of development (Tavazoie and Reid, 2000). The relative percentage of sites at which exclusively $\mathrm{ON}$ - or OFF-center units versus both centertype units were recorded along each electrode penetration was normal (Table 1). As in normal animals, when a mixture of ON and OFF responses was encountered, this always occurred within a limited region (100-300 $\mu \mathrm{m}$ ) adjacent to the optic tract, where the C layers are typically found (Fig. 7) (Stryker and Zahs, 1983). Multiple vertical electrode penetrations were used to map the distribution of responses in each animal. In normal animals, this always reveals four sublaminas in the same sequence: contralateral-ON, contralateral-OFF, ipsilateral-ON, and ipsilateralOFF (Stryker and Zahs, 1983; Zahs and Stryker, 1985). In the epibatidine-recovery animals, there was much more variation in the number of ON or OFF regions within a single penetration; anywhere from two to six distinct $\mathrm{ON}$ or OFF regions were detected. Reconstruction of adjacent penetrations revealed that these $\mathrm{ON}$ or OFF regions were present in varying sequence from one penetration site to the next (Fig. 7). In addition, although eye-specific borders always correspond to receptive field centertype borders in normal animals, regardless of the angle of the electrode penetration (Stryker and Zahs, 1983; Zahs and Stryker, 

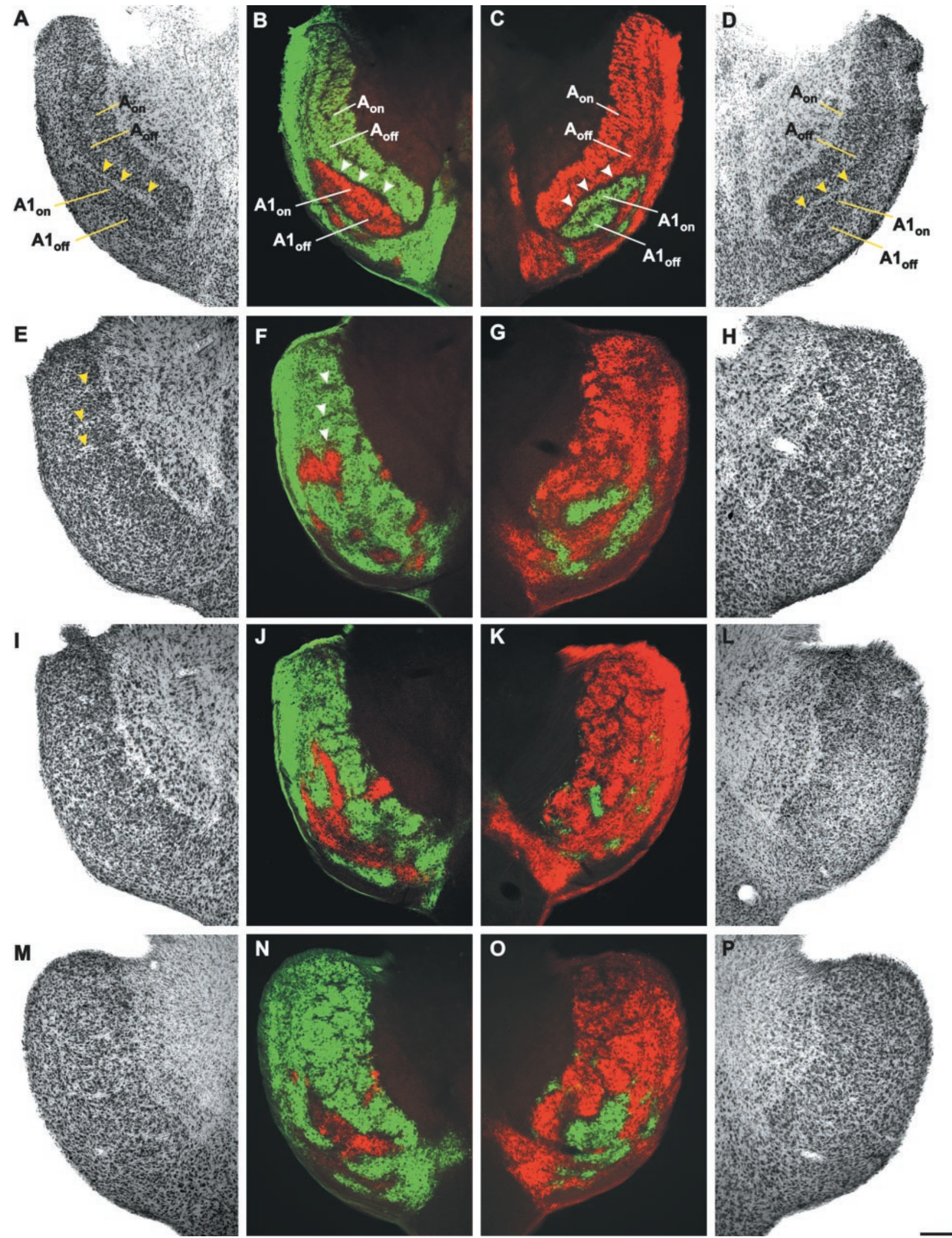

Figure 4. Relationship between afferent projection patterns and cytoarchitecture in epibatidine-recovery animals. $A-D$, Bilateral representation of cytoarchitecture $(A, D)$ and retinal afferents $(B, C)$ to the LGN of a saline-treated P25 ferret; lamina A and A1 and their respective ON $\left(A_{o n}, A 1_{o n}\right)$ and $\operatorname{OFF}\left(A_{o f f}, A 1_{o f f}\right)$ sublaminas are clearly present from the pattern of both afferent and cytoarchitectural labeling (arrowheads indicate the border between layers A and A1, separated by an intralaminar space). $E-P$, LGNs of epibatidine-treated $\mathrm{P} 25+$ ferrets labeled to reveal cytoarchitecture $(E, H, I, L, M$, $P$ ) and retinogeniculate afferents $(F, G, J, K, N, O) . E, F, I, J$, Adjacent sections (50 $\mu \mathrm{m}$ each) through the depth of the same LGN. $M, N, O, P$, Bilateral LGNs from the same animal; arrowheads in $E$ and $F$ indicate ectopic intralaminar spaces. Scale bar, $100 \mu \mathrm{m}$. 

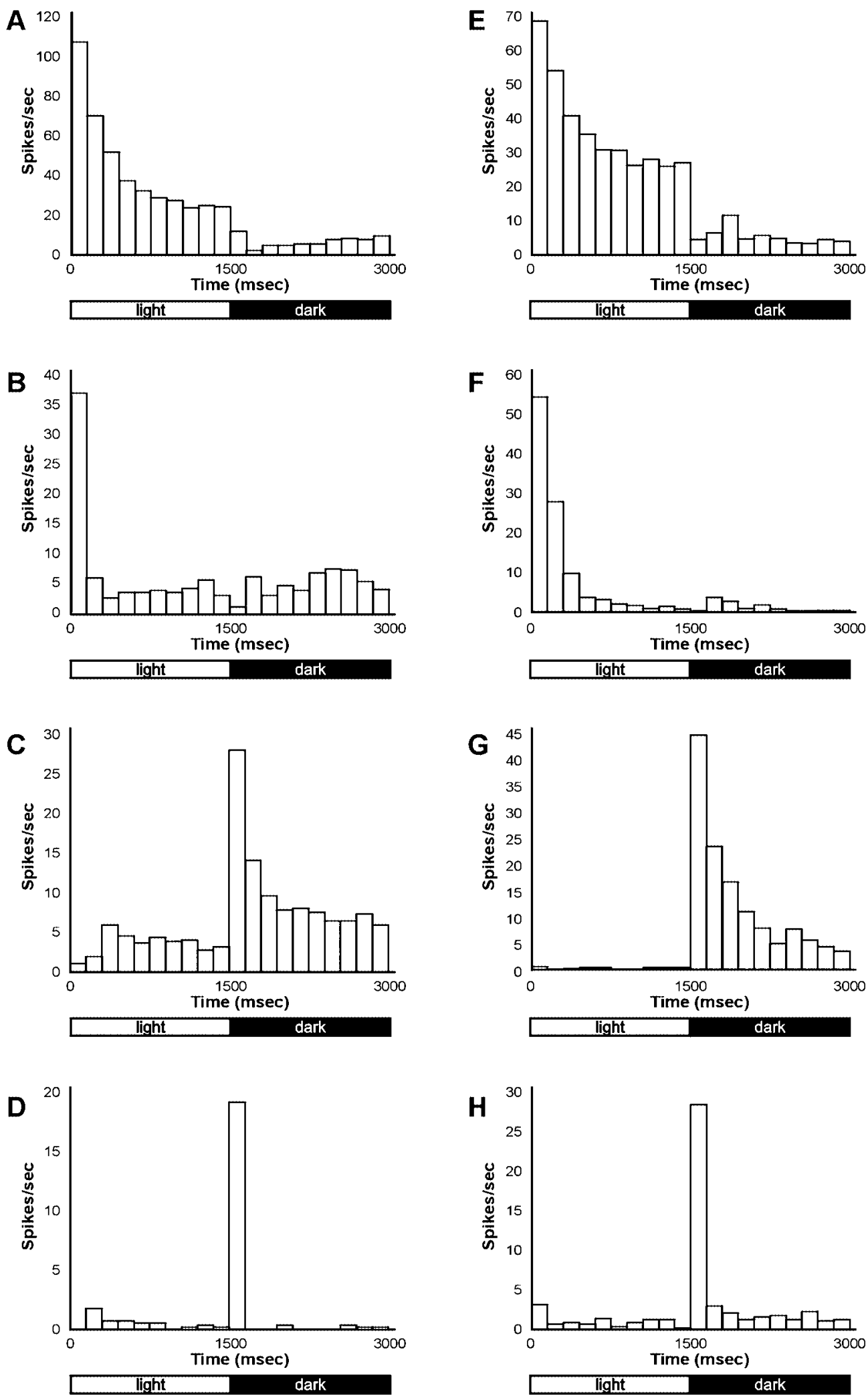

Figure 5. PSTHs showing ON or OFF responses of single LGN neurons from saline-treated $(A-D)$ and epibatidinerecovery $(E-H)$ ferrets. Sustained $(A$, $E)$ and transient $(B, F)$ ON responses and sustained $(C, G)$ and transient $(D$, $H$ ) OFF responses were present in both groups of animals.

1985), in the treated animals, exclusively ON- or OFF-center cells often straddled the boundaries of eye-specific layers (for example, see arrows in Fig. 7). Thus, disrupting the pattern of eye-specific layers disrupts the organization of $\mathrm{ON}$ and OFF sublaminas in the LGN but has little effect on the receptive field properties of the individual cells.
Surprisingly, in the epibatidine-recovery animals, the topographic representation of the binocular visual field was mapped smoothly in a given penetration, even across the boundaries of eye-specific borders (Fig. 8). As in normal ferrets, peripheral azimuths were represented rostrally, whereas higher elevations were represented dorsally (Fig. 8) (Stryker 

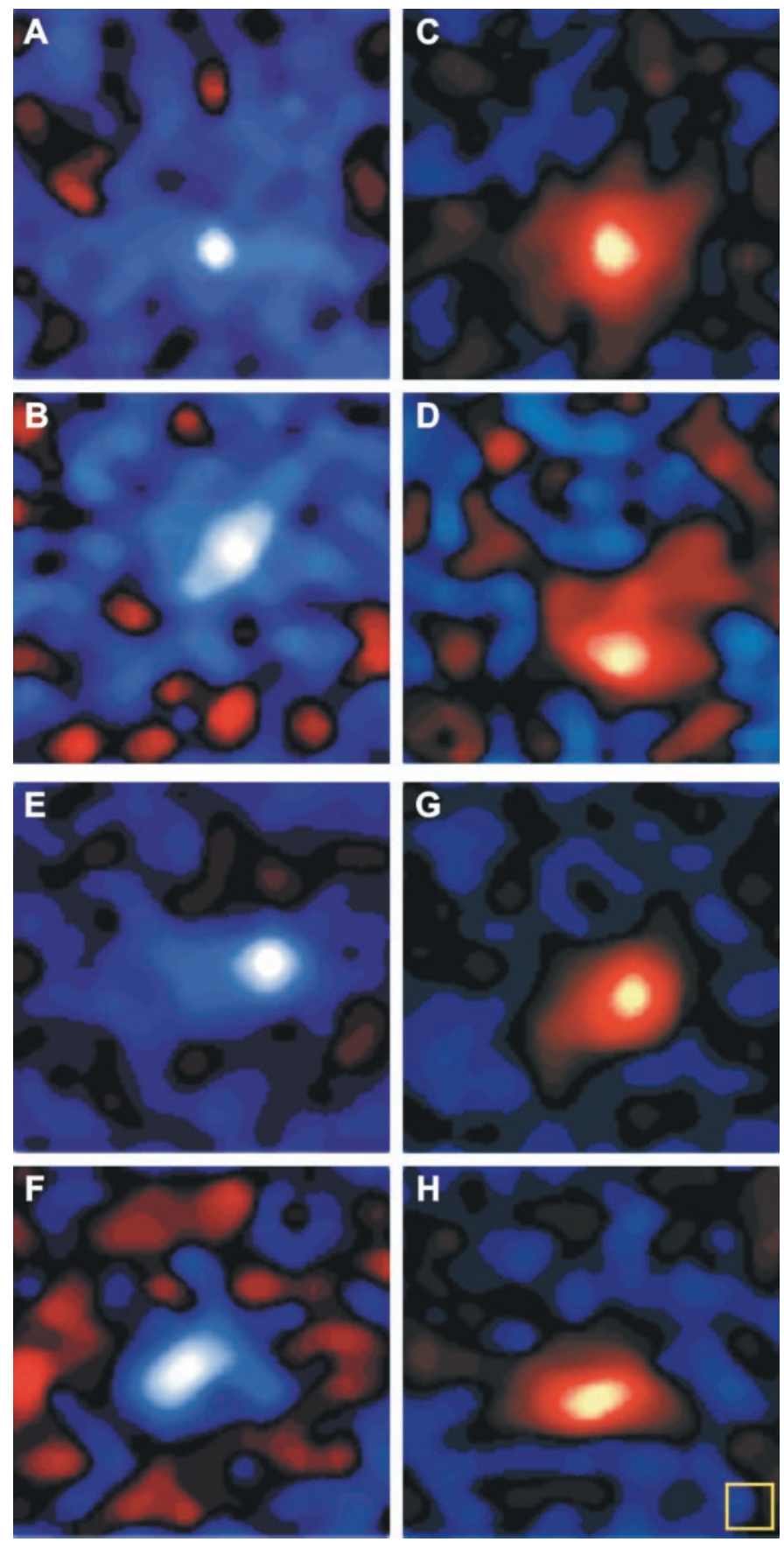

Figure 6. Receptive field organization of LGN neurons in saline-treated $(A-D)$ and epibatidine-recovery $(E-H)$ ferrets. Both OFF- $(A, B, E, F)$ and ON- $(C, D, G, H)$ center surround receptive fields were present in both treatment groups. Areas of the receptive fields of the cells excited by light stimuli (ON subregions) are shown in red, and areas excited by dark stimuli (OFF subregions) are shown in blue. Brightness corresponds to the strength of the responses of the cells. Yellow square, $2 \times 2$ degrees of visual angle.

and Zahs, 1983; Zahs and Stryker, 1985). Thus, dramatically disrupting the organization of eye-specific and ON and OFF lamination does not affect the gross topographic representation of visual space in the LGN. This finding is especially unexpected given the widely varying pattern of eye-specific layers both between and within the LGNs of the treated animals.
Table 1. Relative proportion of sites where exclusively ON- or OFFcenter versus both-center-type inputs were recorded in the LGN

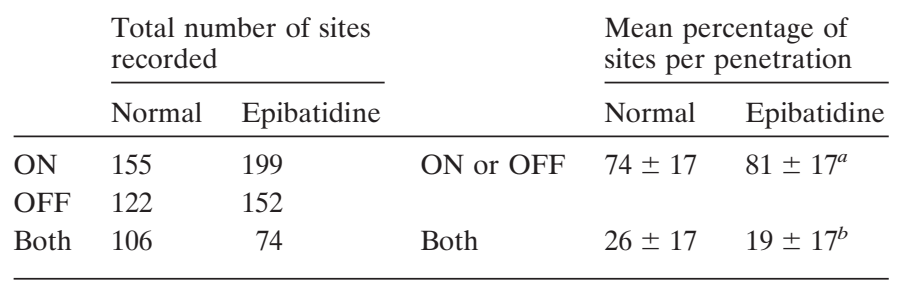

${ }^{a} p=0.16 ; \pm \mathrm{SD}$

${ }^{b} p=0.17 ; \pm \mathrm{SD}$.

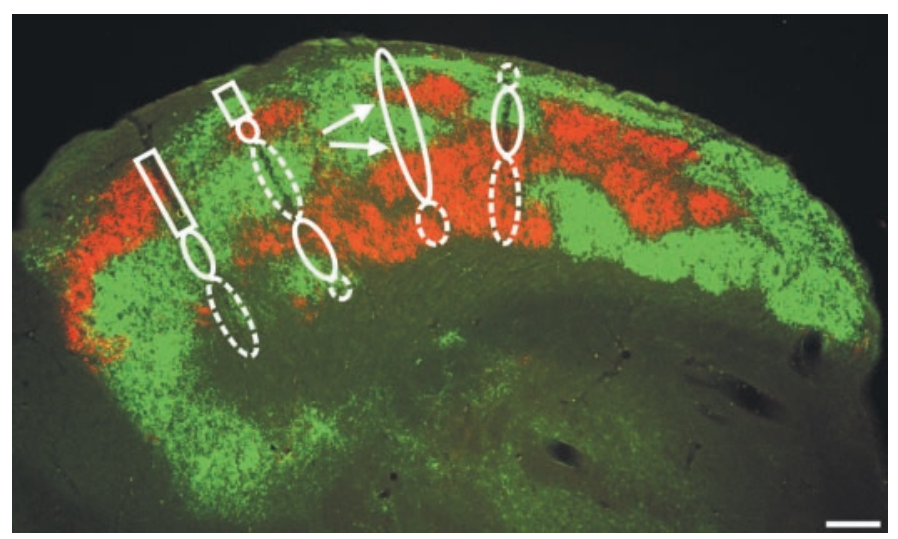

Figure 7. Photomicrograph of the LGN showing the relationship between eye-specific and ON-OFF organization from an epibatidinerecovery ferret. Green represents inputs from the contralateral eye, and red represents inputs from the ipsilateral eye. Four electrode penetrations, spaced $300 \mu \mathrm{m}$ apart across the rostrocaudal axis of the medial portion of the LGN, are shown; responses were recorded every $100 \mu \mathrm{m}$ along each penetration. Solid ovals encompass regions along the electrode track in which cells with ON-center receptive fields were recorded. Dashed ovals encompass regions along the electrode tracks in which cells with OFFcenter receptive fields were recorded. Rectangles encompass regions in which cells with mixed $\mathrm{ON}$ and $\mathrm{OFF}$ receptive fields were recorded. Arrows indicate one case in which a region of ON-center cells traverses several eye-specific borders. Tissue section is in the parasagittal plane; at the age shown (P65), rostral is to the right, and dorsal is to the top of the image. Scale bar, $200 \mu \mathrm{m}$.

\section{DISCUSSION}

Our results show that the development of lamination in the LGN reflects three processes: the segregation of retinogeniculate afferents, the patterning of those afferents into layers, and the emergence of cellular layers that correspond to the pattern of afferent layers. During normal development, the segregation of retinal afferents into eye-specific territories and the patterning of those territories into afferent layers of stereotyped shape, size, and position occur in parallel. Eye-specific afferent layers emerge from a state in which inputs from the two eyes overlap extensively and then segregate directly into layers A and A1; they never segregate into patches within the region of initial overlap (Fig. 1A-C) (Linden et al., 1981; Shatz, 1983; Cucchiaro and Guillery, 1984; Hutchins and Casagrande, 1990; Penn et al., 1998; Hahm et al., 1999). This has suggested that afferent segregation and afferent lamination are the same process. Cellular laminas corresponding to the pattern of afferent layers emerge soon after segregation is complete (Rakic, 1976; Linden et al., 1981; Shatz, 1983; Cucchiaro and Guillery, 1984; Hutchins and Casagrande, 1990). Previous experiments show that removal of one eye early in development results in an expansion of the inputs from the 



Figure 8. Retinotopic organization of the LGN in a saline-treated $(A)$ and an epibatidine-recovery $(B)$ ferret. Receptive fields recorded from two electrode penetrations spaced $300 \mu \mathrm{m}$ apart along the rostrocaudal axis of the LGN are shown for both animals. Numbers indicate the order that cells were recorded in each experiment. Recording sites were $100 \mu \mathrm{m}$ apart along the electrode penetration. Green corresponds to contralateral eye responses, and red corresponds to ipsilateral eye responses. In $A$, receptive fields $1-16$ were recorded in one electrode penetration, whereas receptive fields $20-30$ were recorded in a separate electrode penetration positioned $300 \mu \mathrm{m}$ more rostral. Three additional cells (17-19) were recorded at the bottom of the more caudal penetration, but their responses were too weak to plot reliably. In $B$, receptive fields $1-12$ were recorded in one electrode penetration, whereas receptive fields 20-29 were recorded in a separate electrode penetration positioned $300 \mu \mathrm{m}$ more rostral. Seven additional cells (13-19) were encountered at the top of the more rostral penetration, but their receptive fields were located above and peripheral to the tangent screen, so they could not be carefully plotted; they are not included here.

remaining eye to fill almost the entire LGN and a corresponding loss of eye-specific cellular lamination (Rakic, 1981; Guillery et al., 1985a; Sretavan and Shatz, 1986; Garraghty et al., 1988; Morgan and Thompson, 1993). Moreover, in coat-color mutants in which abnormal retinogeniculate projections develop, the cellular laminas mirror those abnormal projections (Guillery, 1969, 1971; Guillery et al., 1985a). These results, in which the pattern of cellular lamination mirrors that of the afferents, suggested that cellular lamination is driven by the projection pattern of the afferents.

Here we show, however, that eye-specific afferent segregation can develop without normal afferent lamination, demonstrating that these are in fact distinct processes. We also show that when eye-specific afferent segregation is not accompanied by normal afferent lamination, interlaminar zones corresponding to the pattern of inputs from the two eyes failed to develop. Therefore, cellular lamination is also decoupled from afferent segregation.

Our results reveal a critical period for normal development of the LGN. Preventing spontaneous retinal activity during this critical period prevents the formation of normal afferent and cellular layers. This indicates that there is something special about the developmental time window in which retinogeniculate segregation normally occurs for proper patterning of laminas in the LGN. Surprisingly, however, axons from the two eyes remain capable of segregating into nonoverlapping termination zones after the end of this critical period for lamination. Thus, the exact timing of lamination is crucial, but eye-specific segregation can occur even if it begins after the developmental stage in which it normally would be complete. It is unclear what factors are essential for normal lamination of the LGN and why these are limited to the developmental period in which eye-specific segregation normally occurs.

It is possible that the pattern of spontaneous retinal activity present from $\mathrm{P} 1$ to $\mathrm{P} 10$ could be instructive toward development of lamination. The pattern of spontaneous retinal activity is different from P1 to P10, when eye-specific segregation normally results in lamination, than it is from P10 to P25 (Wong et al., 1993), when we show that eye-specific segregation results in patches. However, it is unlikely that activity is sufficient to instruct the development of lamination. First, during the same developmental stage when retinogeniculate afferents segregate into eyespecific laminas, retinal activity also induces contralateral and ipsilateral ganglion cell afferents to segregate into clusters rather than layers in the rostral superior colliculus (Thompson and Holt, 1989). Second, in studies in which retinal inputs were rewired into the medial geniculate nucleus, afferents from the two eyes initially overlapped and then segregated into eye-specific patches instead of layers (Angelucci et al., 1997). Spontaneous retinal activity was normal throughout development in these animals, and yet this did not produce normal lamination. These results indicate that layer formation is controlled by cues that are intrinsic and unique to the LGN, rather than by patterns of retinal activity. Moreover, it is hard to imagine how activity could give rise to highly stereotyped eye-specific layers, because activity is likely to differ across animals, and yet eye-specific laminas in the LGN always form in the same positions as layers of essentially invariant size, shape, and orientation (Figs. 1B, 2A, 4A-D) (Linden et al., 1981; Stryker and Zahs, 1983; Cucchiaro and Guillery, 1984; Zahs and Stryker, 1985; Hutchins and Casagrande, 1990; Penn et al., 1998; Hahm et al., 1999; Stellwagen and Shatz, 2002).

Theoretically, the timing of fiber ingrowth could influence the final pattern of eye-specific layers in the LGN. Axons from the contralateral eye arrive before axons from the ipsilateral eye (Linden et al., 1981; Cucchiaro and Guillery, 1984), raising the possibility that layers are patterned such that earliest arriving axons terminate in the medial-most LGN (future location of layer 
A). However, axons from the two eyes do not segregate as they grow into the LGN; they overlap extensively throughout the LGN early in development (Fig. 1A,C) (Linden et al., 1981; Cucchiaro and Guillery, 1984; Penn et al., 1998). The overlap seen in normal $\mathrm{P} 1$ ferrets is similar to that seen in epibatidine-treated ferrets at P10 (Fig. 1A,C,E) (Penn et al., 1998). Therefore, differences in the timing of ingrowth cannot explain the normal patterning of eye-specific laminas in the LGN or the lack of normal laminas seen in the epibatidine-recovery animals.

Patterning of layers thus almost certainly relies on the presence of signals that bias the location and boundaries of the regions into which afferents from one or the other eye segregate. Recent studies have demonstrated a critical role for the ephrin family of axon guidance cues in directing nasal and temporal retinal axons to different regions in the optic tectum and LGN (for review, see Feldheim et al., 1998; Flanagan and Vanderhaeghen, 1998), making the ephrins premiere candidates for patterning of the contralateral A (nasal-retina derived) and ipsilateral A1 (temporalretina derived) layers in the LGN. However, it is important to note that silencing retinal activity from $\mathrm{P} 1$ to $\mathrm{P} 10$ prevents normal afferent lamination from developing (Fig. 1E) (Penn et al., 1998). Thus, if ephrin-like cues do in fact guide contralateral and ipsilateral retinal afferents to their appropriate regions in the LGN, activity appears to be required to "read out" these cues. Additionally, the eye-specific segregation without normal afferent and cellular lamination seen in the LGN of epibatidine-recovery animals suggests that the ability of guidance cues to direct incoming axons to their appropriate regions in the LGN is restricted to the phase of development when axons normally segregate in this target. This would explain why in the epibatidine-recovery animals, inputs from the two eyes segregate into a variable projection pattern.

Our physiological data show that, just as eye-specific retinogeniculate connections can emerge in the absence of normal eye-specific layers, segregated ON- or OFF-center LGN neurons can develop without accompanying normal ON and OFF sublamination. Nevertheless, disrupting the spatial organization of eyespecific layers appears to have a profound impact on the overall arrangement of ON and OFF segregation in the LGN. This is surprising in light of a previous report showing normal ON-OFF lamination in the expanded projection from the remaining eye of monocularly enucleated animals (Morgan and Thompson, 1993). The disruption of ON and OFF sublaminas in epibatidinerecovery animals occurs despite the fact that they experienced normal patterns of retinal waves from P12 to P25, when the differential activity of ON- versus OFF-center ganglion cells in the retina drives functional segregation of ON and OFF pathways in the LGN (Wong and Oakley, 1996; Bisti et al., 1998; Myhr et al., 2001). Because we did not record from individual ganglion cells in the epibatidine-recovery animals, we cannot be sure that the firing patterns of these neurons are completely normal; thus, we cannot rule out a role for P12-P25 retinal activity in forming normal ON and OFF sublaminas. However, our data suggest that delaying eye-specific segregation until after P10 influences the organization of ON-OFF sublaminas, a feature that, based on its later timing during normal development, had appeared independent of the formation of eye-specific laminas.

Apart from the disruption in $\mathrm{ON}$ and OFF patterning, the physiology and functional organization in the LGN of animals in which afferents are segregated but lamination is disrupted are remarkably normal. The presence of normal retinotopy in these animals indicates that, despite the large degree of overlap in the retinogeniculate projections from each eye at P10 (Fig. 1C,E), axons from ganglion cells in the two eyes that view identical regions in visual space segregated into neighboring domains during the recovery period. This is especially surprising given the highly variable pattern of eye-specific inputs to the LGN of the epibatidine-recovery animals, and it indicates that binocular, retinotopically organized maps of visual space can be organized in many different ways. The relatively normal physiology and functional architecture of the LGN in epibatidine-recovery animals also raises the question of the functional significance of the highly stereotyped LGN lamination in normal animals.

\section{REFERENCES}

Angelucci A, Clasca F, Bricolo E, Cramer KS, Sur M (1997) Experimentally induced retinal projections to the ferret auditory thalamus: development of clustered eye-specific projections in a novel target. J Neurosci 17:2040-2055.

Bisti S, Gargini C, Chalupa LM (1998) Blockade of glutamate-mediated afferent activity in the developing retina perturbs the functional segregation of ON and OFF pathways. J Neurosci 18:5019-5025.

Brunso-Bechtold JK, Casagrande VA (1981) Effect of bilateral enucleation on the development of layers in the dorsal lateral geniculate nucleus. Neuroscience 2:589-597.

Chapman B (2000) Necessity for afferent activity to maintain eyespecific segregation in ferret lateral geniculate nucleus. Science 287:2479-2482.

Chapman B, Gödecke I (2000) Cortical cell orientation selectivity fails to develop in the absence of ON-center ganglion cell activity. J Neurosci 20:1922-1930.

Cucchiaro J, Guillery RW (1984) The development of the retinogeniculate pathways in normal and albino ferrets. Proc R Soc Lond B Biol Sci 223:141-164.

Cusato K, Stagg SB, Reese BE (2001) Two phases of increased cell death in the inner retinal following early elimination of the ganglion cell population. J Comp Neurol 439:440-449.

Feldheim DA, Vanderhaeghen P, Hansen MJ, Frisen J, Lu Q, Barbacid M, Flanagan JG (1998) Topographic guidance labels in a sensory projection to the forebrain. Neuron 21:1303-1313.

Feller MB, Wellis DP, Stellwagen D, Werblin F, Shatz CJ (1996) Requirement for cholinergic synaptic transmission in the propagation of spontaneous retinal waves. Science 272:1182-1187.

Flanagan JG, Vanderhaeghen P (1998) The ephrins and Eph receptors in neural development. Annu Rev Neurosci 21:309-345.

Garraghty PE, Shatz CJ, Sur M (1988) Prenatal disruption of binocular interactions creates novel lamination in the cat's lateral geniculate nucleus. Vis Neurosci 1:93-102.

Guillery RW (1969) An abnormal retinogeniculate projection in Siamese cats. Brain Res 14:739-741.

Guillery RW (1971) An abnormal retinogeniculate projection in the albino ferret (Mustela furo). Brain Res 33:482-485.

Guillery RW, LaMantia AS, Robson JA, Huang K (1985a) The influence of retinal afferents upon the development of layers in the dorsal lateral geniculate nucleus of mustelids. J Neurosci 5:1370-1379.

Guillery RW, Ombrellaro M, LaMantia AL (1985b) The organization of the lateral geniculate nucleus and of the geniculocortical pathway that develops without retinal afferents. Brain Res 352:221-233.

Hahm JO, Cramer KS, Sur M (1999) Pattern formation by retinal afferents in the ferret lateral geniculate nucleus: developmental segregation and the role of $N$-methyl-D-aspartate receptors. J Comp Neurol 411:327-345.

Hanson ES, Reese BE (1993) Rapid plastic response following early retinal lesions in rats. Brain Res Dev Brain Res 73:293-298.

Henderson Z, Finlay BL, Wikler KC (1988) Development of ganglion cell topography in ferret retina. J Neurosci 8:1194-1205.

Hutchins JB, Casagrande VA (1990) Development of the lateral geniculate nucleus: interactions between retinal afferent, cytoarchitectonic, and glial cell process lamination in ferrets and tree shrews. J Comp Neurol 298:113-128.

Jeffrey G (1985) Retinotopic order appears before ocular separation in the developing visual pathways. Nature 313:575-576.

Jones EG (1985) The thalamus. New York: Plenum.

Linden DC, Guillery RW, Cucchiaro J (1981) The dorsal lateral geniculate nucleus of the normal ferret and its postnatal development. J Comp Neurol 203:189-211.

Lund RD, Cunningham TJ, Lund JS (1973) Modified optic projections after unilateral eye removal in young rats. Brain Behav Evol 8:51-72.

Morgan J, Thompson ID (1993) The segregation of ON- and OFFcenter responses in the ferret lateral geniculate nucleus of normal and monocularly enucleated ferrets. Vis Neurosci 10:303-311.

Myhr KL, Lukasiewicz PD, Wong RO (2001) Mechanisms underlying 
developmental changes in the firing patterns of ON and OFF retinal ganglion cells during refinement of their central projections. J Neurosci 21:8664-8671.

Penn AA, Riquelme PA, Feller MB, Shatz CJ (1998) Competition in retinogeniculate patterning driven by spontaneous activity. Science 279:2108-2112.

Rakic P (1976) Prenatal genesis of connections subserving ocular dominance in the rhesus monkey. Nature 261:467-471.

Rakic P (1981) Development of visual centres in the primate brain depends on binocular competition before birth. Science 214:928-931.

Reese BE, Thompson WF, Peduzzi JD (1994) Birthdates of neurons in the retinal ganglion cell layer of the ferret. J Comp Neurol 341:464475.

Reid RC, Victor JD, Shapley RM (1997) The use of m-sequences in the analysis of visual neurons: linear receptive field properties. Vis Neurosci 14:1015-1027.

Rossi FM, Pizzorusso T, Porciatti V, Marubio LM, Maffei L, Changuex JP (2001) Requirement of the nicotinic acetylcholine receptor $\beta 2$ subunit for the anatomical and functional development of the visual system. Proc Natl Acad Sci USA 98:6453-6458.

Shatz CJ (1983) The prenatal development of the cat's retinogeniculate pathway. J Neurosci 3:482-499.

Shatz CJ, Stryker MP (1988) Prenatal tetrodotoxin infusion blocks segregation of retinogeniculate afferents. Science 242:87-89.

Sretavan DW, Shatz CJ (1986) Prenatal development of cat retinogeniculate axon arbors in the absence of binocular interactions. J Neurosci 6:990-1003.
Stellwagen D, Shatz CJ (2002) An instructive role for retinal waves in the development of retinogeniculate connectivity. Neuron 33:357-367.

Stellwagen D, Shatz CJ, Feller MB (1999) Dynamics of retinal waves are controlled by cyclic AMP. Neuron 24:673-685.

Stryker MP, Zahs KR (1983) On and off sublaminae in the lateral geniculate nucleus of the ferret. J Neurosci 10:1943-1951.

Tavazoie SF, Reid RC (2000) Diverse receptive fields in the lateral geniculate nucleus during thalamocortical development. Nat Neurosci 3:608-616.

Thompson I, Holt C (1989) Effects of intraocular tetrodotoxin on the development of the retinocollicular pathway in the Syrian hamster. J Comp Neurol 282:371-388.

Thompson ID, Morgan JE, Henderson Z (1993) The effects of monocular enucleation on ganglion cell number and terminal distribution in the ferret's uncrossed retinal pathway. Eur J Neurosci 5:357-367.

Wong RO, Oakley DM (1996) Changing patterns of spontaneous bursting activity of on and off retinal ganglion cells during development. Neuron 16:1087-1095

Wong RO, Meister M, Shatz CJ (1993) Transient period of correlated bursting activity during development of the mammalian retina. Neuron 11:923-938.

Wong WT, Myhr KL, Miller ED, Wong RO (2000) Developmental changes in the neurotransmitter regulation of correlated spontaneous retinal activity. J Neurosci 20:351-360.

Zahs KR, Stryker MP (1985) The projection of the visual field onto the lateral geniculate nucleus of the ferret. J Comp Neurol 241:210-224. 